\title{
Dynamics of decoherence: universal scaling of the decoherence factor
}

\author{
Sei Suzuki, ${ }^{1}$ Tanay Nag, ${ }^{2}$ and Amit Dutta ${ }^{2}$ \\ ${ }^{1}$ Department of Liberal Arts, Saitama Medical University, Moroyama, Saitama 350-0495, Japan \\ ${ }^{2}$ Department of Physics, Indian Institute of Technology, Kanpur 208 016, India
}

\begin{abstract}
We study the time dependence of the decoherence factor (DF) of a qubit globally coupled to an environmental spin system (ESS) which is driven across the quantum critical point (QCP) by varying a parameter of its Hamiltonian in time $t$ as $1-t / \tau$ or $-t / \tau$, to which the qubit is coupled starting at the time $t \rightarrow-\infty$; here, $\tau$ denotes the inverse quenching rate. In the limit of weak coupling, we analyze the time evolution of the DF in the vicinity of the QCP (chosen to be at $t=0$ ) and define three quantities, namely, the generalized fidelity susceptibility $\chi_{F}(\tau)$ (defined right at the QCP), and the decay constants $\alpha_{1}(\tau)$ and $\alpha_{2}(\tau)$ which dictate the decay of the DF at a small but finite $t(>0)$. Using a dimensional analysis argument based on the Kibble-Zurek healing length, we show that $\chi_{F}(\tau)$ as well as $\alpha_{1}(\tau)$ and $\alpha_{2}(\tau)$ indeed satisfy universal power-law scaling relations with $\tau$ and the exponents are solely determined by the spatial dimensionality of the ESS and the exponents associated with its QCP. Remarkably, using the numerical t-DMRG method, these scaling relations are shown to be valid in both the situations when the ESS is integrable and non-integrable and also for both linear and non-linear variation of the parameter. Furthermore, when an integrable ESS is quenched far away from the QCP, there is a predominant Gaussian decay of the DF with a decay constant which also satisfies a universal scaling relation.
\end{abstract}

PACS numbers:

In the context of quantum computation and information [1, 2], one of the major issues is the study of decoherence [3, 4, namely, the loss of coherence in a quantum system due to its interaction with the environment. To investigate the environment induced decoherence of a qubit in the vicinity of a quantum critical point (QCP) [5, 6] of the environment, a paradigmatic model known as the central spin model (CSM) 7, 8] has been generalized to the context of a quantum phase transition [9].

In the CSM a central spin (CS) or a qubit is globally coupled to an environmental quantum many body system, usually chosen to be a quantum spin system, referred to as the environmental spin system (ESS) in the subsequent discussions. The ESS is initially in its ground state while the CS is in a pure state; the global coupling between the qubit and the environment is so chosen that the subsequent time evolution of the initial ground state wave function of the ESS occurs along two channels dictated by two different Hamiltonians. Even though the qubit is initially in a pure state, it has been shown that it loses its purity (almost completely) 9] when the ESS is close to its quantum critical point (QCP). Question we address in this letter is as follows: what happens when the ESS is slowly driven across its QCP? Is there a universality associated with the dynamically generated decoherence quantified by the decoherence factor (DF) of the CS especially in a limit when the coupling between the CS and the spin chain is weak?

Although we shall consider more generic nonintegrable models in this letter, let us first illustrate the basic idea using the transverse XY chain consisting of $N$ spins [10] as the environmental spin chain; the model is described by the Hamiltonian

$$
H_{E}^{\mathrm{XY}}=-\sum_{i=1}^{N}\left[J_{x} \sigma_{i}^{x} \sigma_{i+1}^{x}+J_{y} \sigma_{i}^{y} \sigma_{i+1}^{y}+h \sigma_{i}^{z}\right]
$$

where $\sigma$ 's are the standard Pauli matrices, $J_{x}\left(J_{y}\right)$ is the ferromagnetic nearest neighbor interactions along the $x(y)$ directions and $h$ is the transverse field; this spin chain is coupled to the spin- $1 / 2$ qubit by a Hamiltonian $H_{S E}$. In the following, we shall set $J_{x}+J_{y}=1$ and the anisotropy in interaction i.e., $J_{x}-J_{y}$ will be denoted by the parameter $\gamma$. The phase diagram and different phase transitions of the model (1) are presented in the Fig. (1).

Let us first assume that the qubit is coupled to the tunable transverse field of Eq. (1) through the Hamiltonian $H_{S E}=-\delta \sum_{i=1}^{N} \sigma_{i}^{z} \sigma_{S}^{z}$, where $\sigma_{i}^{z}$ is the $i$-th spin of the $\mathrm{XY}$ chain and $\sigma_{S}^{z}$ represents that of the qubit with $\delta$ being the coupling strength. [Notably, whenever the qubit is in the up (down) state, the field $h$ of the ESS gets altered to $h-\delta(h+\delta)$.] We choose the qubit to be initially (at $t \rightarrow-\infty)$ in a pure state $\left|\phi_{S}(t \rightarrow-\infty)\right\rangle=c_{1}|\uparrow\rangle+c_{2}|\downarrow\rangle$ with $\left|c_{1}\right|^{2}+\left|c_{2}\right|^{2}=1$, where $|\uparrow\rangle$ and $|\downarrow\rangle$ represent up and down states of the CS, respectively, and the environment is in the ground state $\left|\phi_{E}(t \rightarrow-\infty)\right\rangle=\left|\phi_{g}\right\rangle$. The initial state of the composite Hamiltonian $H_{E}+H_{S E}$, at $t \rightarrow-\infty$, is then given by the direct product $\mid \psi(t \rightarrow$ $-\infty)\rangle=\left|\phi_{S}(t \rightarrow-\infty)\right\rangle \otimes\left|\phi_{g}\right\rangle$. It can be shown that at a later time $t$, the composite wave function is given by $|\psi(t)\rangle=c_{1}|\uparrow\rangle \otimes\left|\phi_{+}(t)\right\rangle+c_{2}|\downarrow\rangle \otimes\left|\phi_{-}(t)\right\rangle$, where $\left|\phi_{ \pm}\right\rangle$are the wavefunctions evolving with the environment Hamiltonian $H_{E}^{\mathrm{XY}}(h \pm \delta)$ given by the Schrödinger equation $i \partial / \partial t\left|\phi_{ \pm}\right\rangle=H_{E}^{\mathrm{XY}}(h \pm \delta)\left|\phi_{ \pm}\right\rangle$. We therefore find that the coupling $\delta$ essentially provides two channels of evolution of the environmental wave function dictated by two trans- 


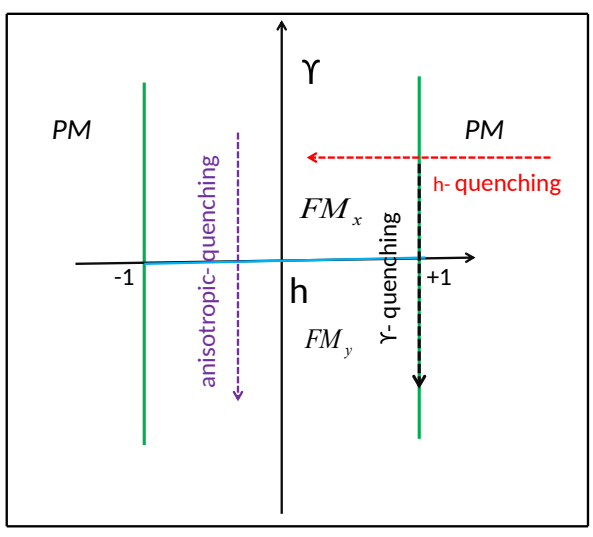

FIG. 1: (Color online) The schematic phase diagram of the transverse XY chain given in Eq. (1) in the $(h-\gamma)$ plane with different quenching paths denoted by arrows. The Ising transition line at $h= \pm 1$ for arbitrary $\gamma$ and the anisotropic transition line at $\gamma=0$ (with $|h|<1$ ) meet at two mulcti-critical points (MCPs) $h= \pm 1$ and $\gamma=0$. When the transverse field $h=1-t / \tau$ is quenched (e.g., setting $\gamma=1$ ) with $t$ from a large positive value to close to the QCP $(t>0)$, one finds $\chi_{F}(\tau) \sim \tau^{1 / 2}, \alpha_{1}(\tau) \sim \tau^{0}$, and $\alpha_{2}(\tau) \sim \tau^{-1 / 2}$ in the limit of small $\delta$; identical scaling relations are obtained when $\gamma$ is quenched across the anisotropic critical line. Finally when $\gamma$ is quenched linearly across the MCP with $h=1$, one finds $\chi_{F}(\tau) \sim \tau^{1 / 3}, \alpha_{1}(\tau) \sim \tau^{-1 / 3}$ and $\alpha_{2} \sim \tau^{-1}$. As shown in the text and in the supplementary material that all these scaling relations are predicted by universal scaling form given in Eq. (4).

verse XY Hamiltonians with the transverse field $h+\delta$ and $h-\delta$, respectively. It is straightforward to show that the DF defined through the relation $D(t)=\left|\left\langle\phi_{+}(t) \mid \phi_{-}(t)\right\rangle\right|^{2}$ measures the purity of the reduced density matrix of the qubit; any non-zero value of $\ln D(t)$ implies that the qubit is in a mixed state. When the transverse field is close to the quantum critical value there is a sharp dip in $D(t)$ which on the one hand, detects the existence of a QCP of the ESS and on the other, establishes that the qubit loses its initial purity almost completely in its vicinity [9].

The quantity $D(t)$ is also known as the Loschmidt echo which has been studied in recent years (both at zero 9 ] and finite temperatures [11]) in the context of decoherence in equilibrium [9, 12, 15] and non-equilibrium situations [16-19] and is closely connected to the dynamical phase transition [20, 21], the statistics of work done 22. and the entropy generation in a quench 23,25$]$.

We assume a weak coupling between the qubit and the environmental Hamiltonian; furthermore the qubit is always globally coupled to time-dependent part of the driven Hamiltonian. Under these circumstances, we now assume the generic situation when a parameter of the ESS Hamiltonian is quenched $\lambda=-t / \tau$, with $t$ starting from a large negative value; here, $\lambda$ is the deviation from

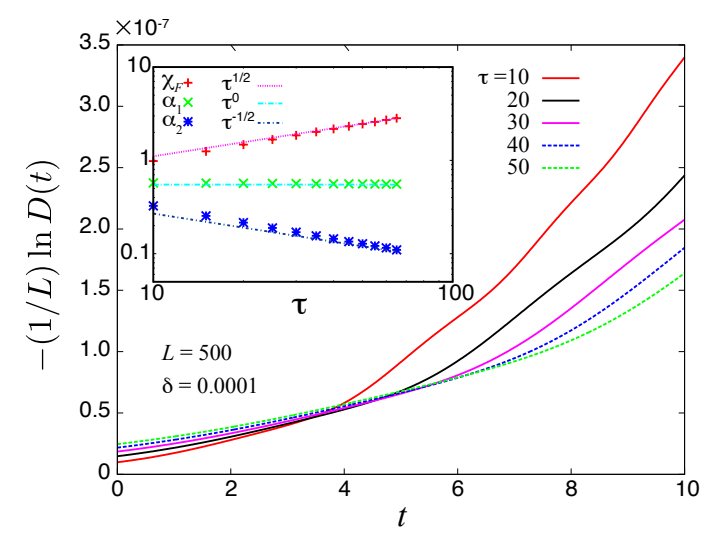

FIG. 2: Time evolution of the decoherence factor for the transverse Ising chain (TIC) as obtained using the t-DMRG method. The transverse field is driven as $h=1-t / \tau$. The scaling of $\chi_{F}(\tau), \alpha_{1}(\tau)$ and $\alpha_{2}(\tau)$ match nicely with the theoretically predicted scaling relations given in Eq. (6).

the QCP (e.g., when the field $h$ in model (1) is quenched, $\lambda=h-1)$, and $\tau(\gg 1)$ is the inverse quenching rate. Consequently, one has two channels of evolutions of the initial ground state of the ESS dictated by two Hamiltonians with parameters $\lambda+\delta$ and $\lambda-\delta$, respectively.

In general, this quantity $D(t)$ can be expanded for sufficiently small $\delta$ and $t$ as

$$
D(t) \approx 1-\left(\chi_{F}(\tau)+\alpha_{1}(\tau) t+\frac{1}{2} \alpha_{2}(\tau) t^{2}\right) \delta^{2} L^{d},
$$

or equivalently,

$$
\frac{1}{L^{d}} \ln D(t) \approx-\left(\chi_{F}(\tau)+\alpha_{1}(\tau) t+\frac{1}{2} \alpha_{2}(\tau) t^{2}\right) \delta^{2},
$$

with

$\chi_{F}(\tau)=-\frac{1}{\delta^{2} L^{d}} \ln D(0), \alpha_{m}(\tau)=-\left.\frac{1}{\delta^{2} L^{d}} \frac{d^{m}}{d t^{m}}(\ln D(t))\right|_{t=0}$,

where $L$ is the linear size of the system. Figure 2 shows the time evolution of $D(t)$ in the transverse Ising chain (TIC) derived from Eq. (1) with $\gamma=1$ (i.e., $J_{y}=0$ ) driven as $h=1-t / \tau$. One can see that $D(t)$ follows Eq. (3) with $\chi_{F}$ and $\alpha_{m}$ changing with $\tau$. It should be noted that there is a prominent Gaussian early-time decay of $D(t)$ (i.e., $\alpha_{1}=0$ ) as predicted in the Ref. [26] only when $D(t)$ is measured between the ground state of unperturbed Hamiltonian; in the present case $\left|\phi_{+}(t=0)\right\rangle \neq\left|\phi_{-}(t=0)\right\rangle$, and hence we indeed have a linear term in Eq. (3) which is also numerically established below and analytically established in the supplementary material (SM).

In this paper, we present the universal scaling of these quantities namely, the quantities $\chi_{F}(\tau), \alpha_{1}(\tau)$ and $\alpha_{2}(\tau)$. We note that $\ln D(\lambda=t=0)=$ 
$\ln \left(\left|\left\langle\phi_{+}(t=0) \mid \phi_{-}(t=0)\right\rangle\right|^{2}\right)$ in fact defines the fidelity 27. between two states evolved from the same initial state along two different channels up to the value $\lambda=0$. In the limit $\delta \rightarrow 0, \ln D(\lambda=t=0)$ can be expanded in the form $\ln D(\lambda=t=0)=-\delta^{2} L^{d} \chi_{F}(\tau)$, where $\chi_{F}(\tau)$ can be viewed as a generalized fidelity susceptibility (see the supplementary material (SM)).

In order to resolve the question raised above concerning the scaling of $\chi_{F}(\tau), \alpha_{1}(\tau)$ and $\alpha_{2}(\tau)$, we resort to dimensional analysis. When the ESS is slowly ramped across its QCP, according to the Kibble-Zurek Scaling (KZS) 28 30] argument, the non-adiabatic effects become prominent only in the vicinity of the QCP; this is a consequence of the diverging relaxation (healing) time at the QCP. As a result, there is a characteristic time scale of the problem $\hat{t} \sim \tau^{\nu z /(\nu z+1)}$ where $\nu$ is the correlation length exponent and $z$ is the dynamical exponent associated with the QCP across which the ESS is driven. This follows from the simple adiabatic impulse argument which relies on the assumption that at $t=\hat{t}$, the rate of change of the Hamiltonian equals the characteristic (healing) time of the problem. So far as low energy modes are concerned, one can assume that within the time window $-\hat{t}<t<\hat{t}$, known as the impulse region, the wave function remains frozen to the adiabatically evolved state at $t=-\hat{t}$, resulting in defects in the final state. One therefore finds a characteristic length scale, known as the healing length $\hat{L}$ that scales as $\hat{L} \sim \tau^{\nu /(\nu z+1)}$; assuming there is one defect within the healing length $\hat{L}$, one immediately finds the KZS of the defect density $n$ in the final state following a slow ramp given by $n \sim 1 / \hat{L}^{d} \sim \tau^{-\nu d /(\nu z+1)}$. We note that the proposed KZS form has been verified and modified in several situations [31 40] (For review see [10, 41, 42]). We shall demonstrate below how the scaling of $\hat{L}$ leads us to the universal scaling of $\chi_{F}(\tau), \alpha_{1}(\tau)$ and $\alpha_{2}(\tau)$ that we want to establish.

To derive the scaling, we first use the definition of the critical exponents $\nu$ and $z$ to find the scaling dimensions $t \sim \hat{L}^{z}$ and $\delta$ (which is equivalent to $\lambda$ ) $\sim \hat{L}^{-1 / \nu}$. Demanding that $\ln D(\lambda=t=0)$ must be dimensionless, we find

$$
\begin{aligned}
\chi_{F}(\tau) & \sim \tau^{(2-d \nu) /(z \nu+1)}, \\
\alpha_{m}(\tau) & \sim \tau^{(2-d \nu-m z \nu) /(z \nu+1)} \quad(m=1,2)
\end{aligned}
$$

Remarkably, the exponents appearing in scaling relations (4) are entirely determined by the universal critical exponents and the spatial dimension $d$. As shown below, these relations hold true for all integrable as well as the nonintegrable situations discussed here. Remarkably, the scaling of $\chi_{F}(\tau)$ also follows from the well established scaling of the fidelity susceptibility associated with the ground state fidelity (see the SM).

Question may arise what would happen if one considers a non-linear drive $\lambda(t)=-|t / \tau|^{r} \operatorname{sign}(t)$ of the ESS where $\operatorname{sign}(t)$ denotes the sign function. In this case, $\hat{L}$ scales as $\tau^{r \nu /(r \nu z+1)}$ [37, 38] and following a similar line of arguments, one finds

$$
\begin{aligned}
\chi_{F}(\tau) & \sim \tau^{r(2-d \nu) /(r z \nu+1)}, \\
\alpha_{m}(\tau) & \sim \tau^{r(2-d \nu-m z \nu) /(r z \nu+1)} \quad(m=1,2) .
\end{aligned}
$$

Equations (4) and (5) contain the central result of our paper.

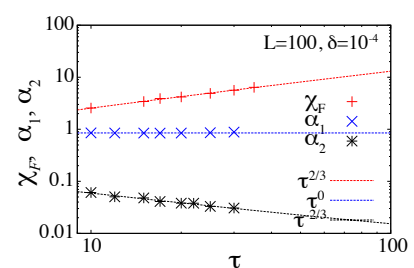

(a)

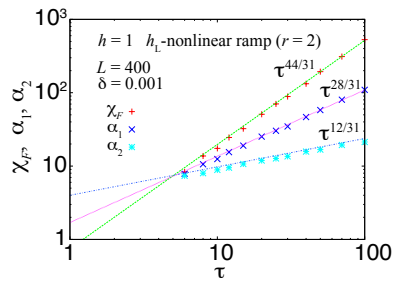

(b)
FIG. 3: (Color online) (a) Scaling of $\chi_{F}, \alpha_{1}$, and $\alpha_{2}$ with respect to $\tau$ for the TIC (Eq. (1) with $J_{y}=0$ ) with a non-linear ramp $(r=2)$ of $h$. We find an very good agreement with analytically predicted scaling in Eq. (7). (b) Scalings of $\chi_{L}, \alpha_{1}$ and $\alpha_{2}$ for the quadratic ramp $(r=2)$ of the longitudinal field in the non-integrable TIC given in 8 with $h=1$; the numerical results as obtained using t-DMRG are in excellent agreement with the predictions as in Eq. 10.

Let us investigate above scaling relations for the integrable model (1) when the transverse field $h$ is quenched as $h(t)=1-t / \tau$ with $J_{y}=0$ and the spin chain is quenched across (but close to) the QCP at $h=1$ (i.e., at $t=0$ ) with exponent $z=\nu=d=1$ : Eqs. (4) and (5) predict:

$$
\chi_{F}(\tau) \sim \tau^{1 / 2}, \quad \alpha_{1}(\tau) \sim \tau^{0}, \quad \alpha_{2}(\tau) \sim \tau^{-1 / 2}
$$

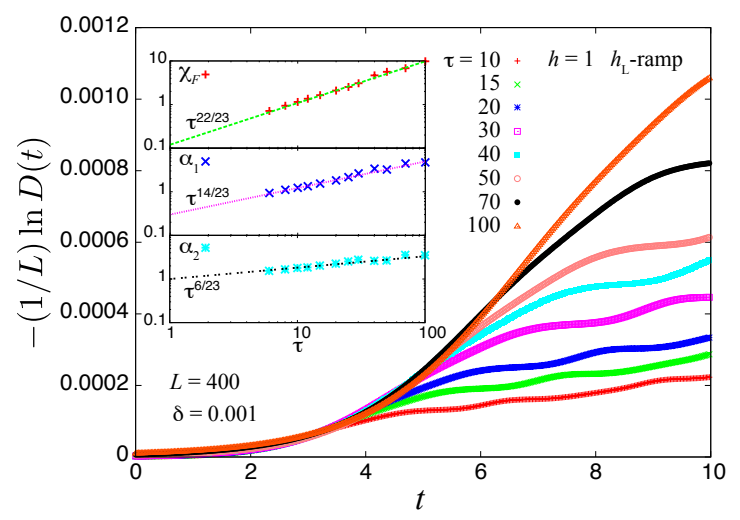

FIG. 4: Early-time evolution of the decoherence factor for the non-integrable TIC in the longitudinal field (8). The transverse field is fixed at $h=1$ and the longitudinal field is ramped as $h_{L}=-t / \tau$. Inset shows scaling of $\chi_{L}, \alpha_{1}$ and $\alpha_{2}$. Results are in good agreement with the prediction (9). 
for the linear ramp, while

$$
\chi_{F}(\tau) \sim \tau^{r /(r+1)}, \quad \alpha_{1}(\tau) \sim \tau^{0}, \quad \alpha_{2}(\tau) \sim \tau^{-r /(r+1)}
$$

for the non-linear ramp. The scaling relations in Eq. (6) has been verified numerically (see Fig. (2)) and established using exact analytical calculations as shown in the SM. Scaling relations for the non-linear ramp presented in Equation (7) is also numerically verified with an excellent agreement as shown in Fig. 3(a).

We now proceed to examine the universal scaling when ESS is non-integrable. We consider the situation when we have a ferromagnetic Ising chain in both transverse and longitudinal fields [43]:

$$
H_{E}^{\mathrm{L}}=-\sum_{i} \sigma_{i}^{z} \sigma_{i+1}^{z}-h_{L} \sum_{i} \sigma_{i}^{z}-\sum_{i} \sigma_{i}^{x}
$$

clearly the Hamiltonian $H_{E}^{\mathrm{L}}$ is at the quantum critical point when $h_{L}=0$ when it represents a critical quantum Ising Hamiltonian obtained by setting $h=J_{x}=1$ and $J_{y}=0$ in Eq. (1). The dynamical exponent $z=1$ and a gap opens up in the spectrum for small non-zero $h_{L}$ as $h_{L}^{8 / 15}$, leading to a value of $\nu=8 / 15$. Considering a similar coupling with the qubit of the form $H_{S E}=$ $-\delta \sum_{i=1}^{N} \sigma_{i}^{z} \sigma_{S}^{z}$ with $\delta \rightarrow 0$, we study the quenching of the ESS close the integrable quantum critical point $\left(h_{L}=0\right)$ by varying $h_{L}=-t / \tau$ starting from a large positive value of $h_{L}$. Scaling relations as obtained from Eqs. (4) and (5) are

$$
\chi_{F}(\tau) \sim \tau^{22 / 23}, \alpha_{1}(\tau) \sim \tau^{14 / 23}, \alpha_{2}(\tau) \sim \tau^{6 / 23},
$$

for the linear ramp and

$$
\begin{aligned}
\chi_{F}(\tau) & \sim \tau^{22 r /(8 r+15)}, \quad \alpha_{1}(\tau) \sim \tau^{14 r /(8 r+15)}, \\
\alpha_{2}(\tau) & \sim \tau^{6 r /(8 r+15)},
\end{aligned}
$$

for the non-linear $\operatorname{ramp} h_{L}(t)=-|t / \tau|^{r} \operatorname{sign}(t)$. Figure 4 shows the early-time behavior of the DF for the model (8) following a linear quenching of $h_{L}$ as obtained using the tDMRG method. The scalings of $\chi_{F}, \alpha_{1}$, and $\alpha_{2}$ extracted close to $t=0$ are good agreement with the prediction in Eq. (9). Figure 3(b), on the other hand, shows the scaling of $\chi_{F}, \alpha_{1}$, and $\alpha_{2}$ for the non-linear ramp; numerical results and theoretical prediction in Eq. 10 with $r=2$ are in excellent agreement.

Let us now address the issue that what happens to the DF when the system is quenched far away from the QCP, i.e., $h$ in model (1) is changed from a large negative to a large positive value. For an integrable model reducible to decoupled two-level problems (like the model (1) or the Kitaev honeycomb model [44]), one can analytically establish that in the limit $t \gg 0, \ln D \sim$ $\left(-t^{2} \delta^{2} L^{d} \tilde{\alpha}_{2}(\tau)\right)$, i.e., there is a prominent Gaussian decay with time [16, 17]. Using the dimensional analysis argument presented above, we immediately conclude that $\tilde{\alpha}_{2}(\tau) \sim \tau^{(2-d \nu-2 z \nu) /(z \nu+1)}$ for a linear quenching of $\lambda$ while for a non-linear $\operatorname{ramp} \tilde{\alpha}_{2}(\tau) \sim \tau^{r(2-d \nu-2 z \nu) /(r z \nu+1)}$. It is noteworthy that whenever $\nu z=1$, the scaling of $\tilde{\alpha}_{2}$ is the same as that of $n$ for both $r=1$ and $r \neq 1$; otherwise, the scaling of $n$ and $\tilde{\alpha}_{2}$ are completely different [17. The universal scaling formula of $\tilde{\alpha}_{2}(\tau)$ explains all the integrable situations discussed in earlier studies. (See the SM for relevant discussion.)

In summary, using the CSM and working in the weak coupling limit, we have provided a universal scaling relation of the generalized fidelity susceptibility $\chi_{F}(\tau)$ as well as the decay constants $\alpha_{1}(\tau)$ and $\alpha_{2}(\tau)$ with the inverse rate $\tau$ in the vicinity of the QCP across which the ESS is driven. We have used a dimensional analysis arguments based on the healing length $\hat{L}$. Remarkably, the exponents are solely determined by the spatial dimension of the ESS and the associated critical exponents. These scaling relations are verified for both integrable and non-integrable models as well as for linear and non-linear ramps. Additionally, we have also derived a universal scaling relation for the decay constant $\tilde{\alpha}_{2}(\tau)$ describing the Gaussian decay of the DF with time when an integrable ESS is quenched far away from the QCP. We reiterate that the DF is equivalent to the Loschmidt echo and the latter has been experimentally observed in the TIC using NMR technique [45]. Therefore an experimental test of our scaling theory on the DF can be realized in a large scale NMR quantum simulator. Furthermore, Kibble-Zurek scaling is already being explored in optical lattices 46.

TN and AD acknowledge Uma Divakaran for fruitful discussions. AD acknowledge SERB, DST India for financial support.

[1] M. A. Nielsen and I. L. Chuang, Quantum Computation and Quantum Information, (Cambridge University Press, Cambridge, UK, 2000).

[2] V. Vedral, Introduction to Quantum Information Science (Oxford University Press, Oxford, UK, 2007).

[3] W. H. Zurek, Rev. Mod. Phys. 75, 715 (2003).

[4] E. Joos, H. D. Zeh, C. Keifer, D. Giulliani, J. Kupsch and I. -O. Stamatescu, Decoherence and appearance of a classical world in a quantum theory, (Springer Press, Berlin) (2003).

[5] S. Sachdev, Quantum Phase Transitions(Cambridge University Press, Cambridge, England,1999).

[6] S. Suzuki, J-i Inoue and Bikas K. Chkarabarti, Quantum Ising Phases and Transitions in Transverse Ising Models (Springer, Lecture Notes in Physics, Vol. 862 (2013)).

[7] John Schliemann, Alexander V. Khaetskii, and Daniel Loss, Phys. Rev. B 66, 245303 (2002).

[8] F. M. Cucchietti, J. P. Paz, and W. H. Zurek, Phys. Rev. A 72, 052113 (2005).

[9] H.T. Quan, Z. Song, X.F. Liu, P. Zanardi, and C.P. Sun, Phys.Rev.Lett. 96, 140604 (2006).

[10] A. Dutta, G. Aeppli, B. K. Chakrabarti, U. Divakaran, T. Rosenbaum and D. Sen, Quantum Phase Transitions 
in Transverse Field Spin Models: From Statistical Physics to Quantum Information (Cambridge University Press, Cambridge, 2015).

[11] P. Zanardi. H. T. Quan, X. Wang and C. P. Sun, Phys. Rev. A 75, 032109 (2007).

[12] S. Sharma, V. Mukherjee, and A. Dutta, Eur. Phys. J. B, 85, 143 (2012).

[13] D. Rossini, T. Calarco, V. Giovannetti, S. Montangero, R. Fazio, Phys. Rev. A 75, 032333 (2007).

[14] F. M. Cucchietti, et al, Phys. Rev. A 75, 032337 (2007); C. Cormick and J. P. Paz, Phys. Rev. A 77, 022317 (2008).

[15] Lorenzo C Venuti and P. Zanardi, Phys. Rev. A 81, 022113 (2010); Lorenzo C. Venuti, N. T. Jacobson, S. Santra, and P. Zanardi, Phys. Rev. Lett. 107, 010403 (2011).

[16] B. Damski, H. T. Quan, and W. H. Zurek Phys. Rev. A 83, 062104 (2011).

[17] T. Nag, U. Divakaran and A. Dutta, Phys. Rev. B 86, 020401 (R) (2012).

[18] V. Mukherjee, S. Sharma, A. Dutta, Phys. Rev. B 86, 020301 (R) (2012).

[19] S. Sharma, A. Russomanno, G. E. Santoro and A. Dutta, EPL 106, 67003 (2014)

[20] M. Heyl, A. Polkovnikov, and S. Kehrein, Phys. Rev. Lett., 110, 135704 (2013).

[21] S. Sharma, S. Suzuki and A. Dutta, arXiv:1506.00477 (2015) (to appear in Phys. Rev. B (2015)).

[22] A. Gambassi and A. Silva, arXiv: 1106.2671 (2011); P. Smacchia and A. Silva, Phys. Rev. E 88, 042109, (2013).

[23] R. Dorner, J. Goold, C. Cormick, M. Paternostro and V. Vedral, Phys. Rev. Lett. 109, 160601 (2012).

[24] S. Sharma and A. Dutta, Phys. Rev. E 92, 022108 (2015).

[25] A. Russomanno, S. Sharma, A. Dutta, G. E. Santoro, J. Stat. Mech. P08030, (2015).

[26] A. Peres, Phys. Rev. A 30, 1610 (1984).

[27] We emphasize that the fidelity defined here should not be confused with the ground state quantum fidelity as elaborated in the SM.

[28] T. W. B. Kibble, J. Phys. A 9, 1387 (1976), and Phys. Rep. 67, 183 (1980); W. H. Zurek, Nature (London) 317,
505 (1985), and Phys. Rep. 276, 177 (1996).

[29] W. H. Zurek, U. Dorner, and P. Zoller, Phys. Rev. Lett. 95, 105701 (2005).

[30] A. Polkovnikov, Phys. Rev. B 72, 161201(R) (2005).

[31] B. Damski, Phys. Rev. Lett. 95, 035701 (2005).

[32] J. Dziarmaga, Phys. Rev. Lett. 95, 245701 (2005).

[33] R. W. Cherng and L. S. Levitov, Phys. Rev. A 73, 043614 (2006).

[34] V. Mukherjee, U. Divakaran, A. Dutta, and D. Sen, Phys. Rev. B 76, 174303 (2007); U. Divakaran, V. Mukherjee, A. Dutta, and D. Sen, J. Stat. Mech. P02007 (2009); V. Mukherjee and A. Dutta, EPL 92 , 37004 (2010).

[35] K. Sengupta, D. Sen, and S. Mondal, Phys. Rev. Lett. 100, 077204 (2008); D. Sen, K. Sengupta, S. Mondal, Phys. Rev. Lett. 101, 016806 (2008).

[36] A. Dutta, R. R. P. Singh, and U. Divakaran, EPL 89, 67001 (2010); T. Hikichi, S. Suzuki, and K. Sengupta, Phys. Rev. B 82, 174305 (2010).

[37] Diptiman Sen, et al, Phys. Rev. Lett. 101, 016806 (2008). S. Mondal, K. Sengupta and Diptiman Sen, Phys. Rev. B 79, 045128 (2009).

[38] R. Barankov and A. Polkovnikov, Phys. Rev. Lett. 101, 076801 (2008).

[39] S.Deng, G. Ortiz, L. Viola EPL, 84, 67008 (2008).

[40] U. Divakaran, A. Dutta, and D. Sen, Phys. Rev. B 78, 144301 (2008).

[41] A. Polkovnikov, K. Sengupta, A. Silva, and M. Vengalattore, Rev. Mod. Phys. 83, 863 (2011).

[42] J. Dziarmaga, Advances in Physics 59, 1063 (2010).

[43] F. Pollmann, S. Mukerjee, A. G. Green, and J. E. Moore, Phys. Rev. E 81, 020101(R) (2010).

[44] A. Kitaev, Ann. Phys. 321, 2 (2006).

[45] J. Zhang, F. M. Cucchietti, C. M. Chandrashekar, M. Laforest, C. A. Ryan, M. Ditty, A. Hubbard, J. K. Gamble, and R. Laflamme, Phys. Rev. A 79, 012305 (2009).

[46] S. Braun, M. Friesdorf, S. S. Hodgman, M. Schreiber, J. P. Ronzheimer, A. Riera, M. del Rey, I. Bloch, J. Eisert, U. Schneider, PNAS 112, 3641 (2015)

\title{
Supplementary Material on "Dynamics of decoherence: universal scaling of the decoherence factor"
}

\author{
Sei Suzuki ${ }^{1}$, Tanay Nag ${ }^{2}$, and Amit Dutta ${ }^{2}$ \\ ${ }^{1}$ Department of Liberal Arts, Saitama Medical University, Moroyama, Saitama 350-0495, Japan \\ ${ }^{2}$ Indian Institute of Technology Kanpur, Kanpur 208 016, India
}

In this supplementary material, we shall illustrate how to justify the expansion of $\ln D(t)$ close to $t=0$ and derive the scaling of $\chi_{F}(\tau), \alpha_{1}(\tau)$ and $\alpha_{2}(\tau)$ for a transverse Ising chain using scaling arguments and exact analytical calculations.

In order to estimate the DF in the limit $t \rightarrow 0+$, we first define the generalized time-dependent fidelity susceptibility $\tilde{\chi}_{F}(t)$ as

$$
\tilde{\chi}_{F}(t)=-\left.\frac{1}{\delta^{2} L} \ln D(t)\right|_{\delta \rightarrow 0}
$$

and expend this quantity into Taylor series of $t$ as follows:

$$
\tilde{\chi}_{F}(t)=\chi_{F}(\tau)+\alpha_{1}(\tau) t+\frac{1}{2} \alpha_{2}(\tau) t^{2}+\cdots
$$


Therefore, the DF in the limit $t \rightarrow 0+$ comes out to be

$$
\begin{aligned}
D(t) & =\left|\left\langle\phi_{+}(t) \mid \phi_{-}(t)\right\rangle\right|^{2} \simeq 1-\delta^{2} L \tilde{\chi}_{F}(t) \\
& \simeq 1-\delta^{2} L\left[\chi_{F}(\tau)+\alpha_{1}(\tau) t+\frac{1}{2} \alpha_{2}(\tau) t^{2}\right] .
\end{aligned}
$$

In the following, we shall derive the scaling of $\chi_{F}(\tau), \alpha_{1}(\tau)$ and $\alpha_{2}(\tau)$ in integrable situations.

\section{THE SCALING OF $\chi_{F}(\tau), \alpha_{1}(\tau)$ AND $\alpha_{2}(\tau)$ FOR INTEGRABLE SITUATIONS}

We shall first derive the scaling of $\chi_{F}(\tau)$ or the generalized fidelity susceptibility using the established scaling of the ground state quantum fidelity. As discussed in the main text, let us assume a linear quenching scheme $\lambda=-t / \tau$ (for the quenching of the transverse field $h$ in the transverse Ising model, $\lambda=h-1)$ which stops at the critical point at $\lambda=t=0$. We then calculate the logarithm of the overlap or fidelity, $\ln D(\lambda=t=0)=\ln \left(\left|\left\langle\phi_{+}(t=0) \mid \phi_{-}(t=0)\right\rangle\right|\right)$; expanding up to the second order in $\delta$, one finds $\ln \left(\left|\left\langle\phi_{+}(t=0) \mid \phi_{-}(t=0)\right\rangle\right|\right) \sim-\delta^{2} L^{d} \chi_{F}(\tau)$. It is well established that at the QCP the ground state quantum fidelity susceptibility scales as $L^{2 / \nu-d}$ [S1], where $\nu$ is the correlation length exponent, $d$ is the spatial dimension of the system, and $L$ is the linear size of the system. In the present problem of slow ramp, the characteristic length scale, as discussed in the main text, is given by the healing length $\hat{L} \sim \tau^{\nu /(\nu z+1)}$. Using $\hat{L}$ in the scaling of the ground state fidelity susceptibility one arrives at the scaling relation $\chi_{F}(\tau) \sim \tau^{(2-\nu d) /(\nu z+1)}$, which has been obtained in the main text using a dimensional analysis approach. We note that for this scaling to be valid, one must have $\delta^{-\nu} \gg L \gg \hat{L}$.

We shall illustrate the above scaling considering the environmental Hamiltonian to be a transverse XY chain

$$
H_{\mathrm{E}}=-\sum_{i=1}^{L}\left(J_{x} \sigma_{i}^{x} \sigma_{i+1}^{x}+J_{y} \sigma_{i}^{y} \sigma_{i+1}^{y}+h(t) \sigma_{i}^{z}\right)
$$

and we first consider the Ising limit obtained by setting $J_{y}=0$; we also choose $J_{x}=1$. The coupling Hamiltonian between the system and the environment is given by

$$
H_{\mathrm{SE}}=-\delta \sum_{i=1}^{L} \sigma_{i}^{z} \sigma_{S}^{z}
$$

where $\sigma_{i}^{z}$ is the $i$-spin of the environment chain and $\sigma_{S}^{z}$ represents the spin of the system. Hereafter we assume a linear ramp of the transverse field:

$$
\lambda(t)=h(t)-1=-\frac{t}{\tau}
$$

where $\tau$ stands for the inverse rate of a ramp.

According to the exact solution presented in Ref.[S2] for $L \rightarrow \infty$, the decoherence factor is given by

$$
\begin{aligned}
& D(t) \approx \exp \left(-\frac{L}{2 \pi} \int_{0}^{\pi} d k \ln F_{k}^{-1}(t)\right), \\
& F_{k}(t)=\left|u_{k}^{+*}(t) u_{k}^{-}(t)+v_{k}^{+*}(t) v_{k}^{-}(t)\right|^{2},
\end{aligned}
$$

where $u_{k}^{ \pm}$and $v_{k}^{ \pm}$are the solutions of the time-dependent Bogoliubov-de Gennes equations

$$
\begin{aligned}
i \frac{d}{d t} u_{k}^{ \pm} & =2(h(t) \pm \delta-\cos k) u_{k}^{ \pm}+2 \sin k v_{k}^{ \pm} \\
i \frac{d}{d t} v_{k}^{ \pm} & =2 \sin k u_{k}^{ \pm}-2(h(t) \pm \delta-\cos k) v_{k}^{ \pm}
\end{aligned}
$$

Using the notation, $t_{ \pm}^{\prime}=-4 \tau \sin k(h(t) \pm \delta-\cos k), \tau^{\prime}=4 \tau \sin ^{2} k, z_{ \pm}=t_{ \pm}^{\prime} e^{i \pi / 4} / \sqrt{\tau^{\prime}}$ and $n=-i \tau^{\prime} / 4$, the exact solution of the above equations under the initial conditions $u_{k}^{ \pm}(-\infty)=1$ and $v_{k}^{ \pm}(-\infty)=0$ is obtained as

$$
\begin{aligned}
u_{k}^{ \pm}(t)= & e^{-\pi \tau^{\prime} / 16} e^{i \pi / 4} \\
& \times\left[(1+n) \mathcal{D}_{-n-2}\left(i z_{ \pm}\right)+i z_{ \pm} \mathcal{D}_{-n-1}\left(i z_{ \pm}\right)\right]
\end{aligned}
$$




$$
v_{k}^{ \pm}(t)=\frac{\sqrt{\tau^{\prime}}}{2} e^{-\pi \tau^{\prime} / 16} \mathcal{D}_{-n-1}\left(i z_{ \pm}\right)
$$

where $\mathcal{D}_{m}(i z)$ is the Weber's parabolic cylinder function. We note that $F_{k}(t)=1$ when $\delta=0$, because $F_{k}(t)$ reduces to the norm of the wavefunction.

Hereafter we fix $t=0$ so that $h(t)=1$ and assume $\tau \gg 1$. Under this assumption, one can show that only the long wave lengths with $k \lesssim \tau^{-1 / 2} \ll 1$ give contribution to the integral of $\ln F_{k}^{-1}$ in Eq. S7]. Therefore, in the present situation, one has $t_{ \pm}^{\prime} \approx-4 \tau k\left( \pm \delta+k^{2} / 2\right), \tau^{\prime} \approx 4 \tau k^{2}, z_{ \pm} \approx-2 \sqrt{\tau}\left( \pm \delta-k^{2} / 2\right)$, and $n \approx-i \tau k^{2}$.

Let us consider the situation where $\delta \ll \tau^{-1 / 2} \ll 1$. Noting that $u_{k}^{ \pm}$and $v_{k}^{ \pm}$depend only on $\tau^{\prime}, z_{ \pm}$and $n$, one can see that $\ln F_{k}^{-1}(0)$ is given as a function of $\tau^{1 / 2} k, \tau^{1 / 2} k^{2}$ and $\tau^{1 / 2} \delta$. Therefore the Taylor expansion of $\ln F_{k}^{-1}(0)$ up to the order of $\tau \delta^{2}$ leads to $\ln F_{k}^{-1}(0) \approx \phi\left(\tau^{1 / 2} k, \tau^{1 / 2} k^{2}\right) \tau \delta^{2}$, where $\phi(x, y)$ is a function of $x$ and $y$. We remark here that the linear term of $\delta$ vanishes since $F_{k}$ is an even function of $\delta$. To carry out the integral of $\ln F_{k}^{-1}$, one can safely shifts the upper limit to infinity because there is no contribution to the integral from $k \gtrsim \tau^{-1 / 2}$. Consequently, one obtains

$$
\begin{aligned}
-\frac{1}{L} \ln D(\lambda=t=0) & \sim \int_{0}^{\infty} d k \phi\left(\tau^{1 / 2} k, \tau^{1 / 2} k^{2}\right) \tau \delta^{2} \\
& \sim \tau^{1 / 2} \delta^{2} \int_{0}^{\infty} d \tilde{k} \phi\left(\tilde{k}, \tau^{-1 / 2} \tilde{k}^{2}\right) \\
& \sim \tau^{1 / 2} \delta^{2}\left\{\int_{0}^{\infty} d \tilde{k} \phi(\tilde{k}, 0)+\mathcal{O}\left(\tau^{-1 / 2}\right)\right\} \\
& \sim \tau^{1 / 2} \delta^{2}
\end{aligned}
$$

which gives $\chi_{F}(\tau)=-\ln D(\lambda=t=0) /\left(L \delta^{2}\right) \sim \tau^{1 / 2}$. Noting that the critical exponent $\nu$ associated with the QCP at $h=1$ is $\nu=1$ and spatial dimensionality $d=1$, this scaling is in perfect congruence with the expected universal scaling behavior of $\chi_{F}$. In non-linear ramping case, $\lambda=-|t / \tau|^{r} \operatorname{sign}(t)$, we can proceed using a similar arguments and establish that $\chi_{F}(\tau) \sim \tau^{r /(r+1)}$.

Now, we shall calculate $F_{k}(t)$ explicitly by expanding the Weber function, $\mathcal{D}_{n}(z)$, near $z=0$. One can show that $z \ll 1$ demands $t_{ \pm}^{\prime} \ll \sqrt{\tau^{\prime}}$. We work in the limit of $\delta^{2} \tau \ll 1$. The power series expansion of $\mathcal{D}_{n}(z)$ for small $z$ is given by

$$
\mathcal{D}_{n}(z)=\frac{2^{\frac{n}{2}} \sqrt{\pi}}{\Gamma\left(\frac{1}{2}-\frac{n}{2}\right)}-\frac{2^{\frac{n+1}{2}} \sqrt{\pi} z}{\Gamma\left(\frac{-n}{2}\right)}-\frac{2^{-2+\frac{n}{2}} \sqrt{\pi}(1+2 n) z^{2}}{\Gamma\left(\frac{1}{2}-\frac{n}{2}\right)}
$$

The solution of time-dependent Bogoliubov-de Gennes equations for $t=\epsilon$ are given by

$$
\begin{aligned}
& u_{k}^{ \pm}(t) \simeq e^{-\pi \tau^{\prime} / 16} e^{i \pi / 4}\left[\frac{2^{-\frac{n}{2}} \sqrt{\pi}}{\Gamma\left(\frac{1}{2}+\frac{n}{2}\right)}-\frac{\tau^{\prime} z_{ \pm} \sqrt{\pi} 2^{-\frac{n}{2}}}{4 \sqrt{2} \Gamma\left(1+\frac{n}{2}\right)}+\frac{(1-2 n) z_{ \pm}^{2} \sqrt{\pi} 2^{-\frac{n}{2}}}{\Gamma\left(\frac{1}{2}+\frac{n}{2}\right)}\right] \\
& v_{k}^{ \pm}(t) \simeq \frac{\sqrt{\tau^{\prime}}}{2} e^{-\pi \tau^{\prime} / 16} 2^{-\frac{n}{2}} \sqrt{\frac{\pi}{2}}\left[\frac{1}{\Gamma\left(1+\frac{n}{2}\right)}-\frac{\sqrt{2} i z_{ \pm}}{\Gamma\left(\frac{1}{2}+\frac{n}{2}\right)}-\frac{(1+2 n) z_{ \pm}^{2}}{4 \Gamma\left(1+\frac{n}{2}\right)}\right]
\end{aligned}
$$

where $z_{ \pm}$is defined by $z_{ \pm}=\left(t_{ \pm}^{\prime} / \sqrt{\tau^{\prime}}\right) e^{i \pi / 4}$. Using the above expressions one can find out the quantity $u_{k}^{+*}(t) u_{k}^{-}(t)+$ $v_{k}^{+*}(t) v_{k}^{-}(t)$ which is given by

$$
\begin{aligned}
& u_{k}^{+*}(t) u_{k}^{-}(t)+v_{k}^{+*}(t) v_{k}^{-}(t) \simeq 1+e^{-\pi \tau^{\prime} / 8}\left[z_{+}^{*} a_{1}+z_{-} a_{2}+z_{+}^{*} z_{-} a_{3}+\left(z_{+}^{*}\right)^{2} a_{4}+z_{-}^{2} a_{5}+z_{+}^{*} z_{-}^{2} a_{6}+\left(z_{+}^{*}\right)^{2} z_{-} a_{7}\right] \\
& +O\left(z_{ \pm}^{4}\right)
\end{aligned}
$$


where

$$
\begin{aligned}
& a_{1}=\frac{i \pi \tau^{\prime} \sqrt{2}}{8 \Gamma\left(\frac{1}{2}+\frac{n^{*}}{2}\right) \Gamma\left(1+\frac{n}{2}\right)}-\frac{\pi \tau^{\prime} \sqrt{2}}{8 \Gamma\left(\frac{1}{2}+\frac{n}{2}\right) \Gamma\left(1+\frac{n^{*}}{2}\right)}, \\
& a_{2}=a_{1}^{*}, \\
& a_{3}=\frac{\pi \tau^{\prime}}{4\left|\Gamma\left(\frac{1}{2}+\frac{n}{2}\right)\right|^{2}}+\frac{\pi \tau^{\prime 2}}{32\left|\Gamma\left(1+\frac{n}{2}\right)\right|^{2}}, \\
& a_{4}=-\frac{\pi \tau^{\prime}\left(1+2 n^{*}\right)}{32\left|\Gamma\left(1+\frac{n}{2}\right)\right|^{2}}+\frac{\pi\left(1-2 n^{*}\right)}{\left|\Gamma\left(\frac{1}{2}+\frac{n}{2}\right)\right|^{2}}, \\
& a_{5}=a_{4}^{*}, \\
& a_{6}=-\frac{i \pi \tau^{\prime}(1+2 n) \sqrt{2}}{32 \Gamma\left(\frac{1}{2}+\frac{n^{*}}{2}\right) \Gamma\left(1+\frac{n}{2}\right)}-\frac{\pi \tau^{\prime}(1-2 n)}{4 \sqrt{2} \Gamma\left(\frac{1}{2}+\frac{n}{2}\right) \Gamma\left(1+\frac{n^{*}}{2}\right)}, \\
& a_{7}=a_{6}^{*} .
\end{aligned}
$$

Therefore, $F_{k}(t)$ is given by $F_{k}(t)=1+A_{k}(t)+B_{k}(t)+C_{k}(t)+D_{k}(t)$, where

$$
\begin{aligned}
A_{k}(t) & =2 e^{-\pi \tau^{\prime} / 8}\left[\left|\operatorname{Re}\left(z_{+} a_{1}^{*}\right)\right|+\left|\operatorname{Re}\left(z_{-} a_{2}\right)\right|\right] \\
B_{k}(t) & =2 e^{-\pi \tau^{\prime} / 8}\left[\left|\operatorname{Re}\left(z_{+}^{*} z_{-} a_{3}\right)\right|+\left|\operatorname{Re}\left(z_{+}^{2} a_{4}^{*}\right)\right|+\left|\operatorname{Re}\left(z_{-}^{2} a_{5}\right)\right|\right]+e^{-\pi \tau^{\prime} / 4}\left[2\left|\operatorname{Re}\left(z_{-} z_{+} a_{2} a_{1}^{*}\right)\right|+\left|z_{+}\right|^{2}\left|a_{1}\right|^{2}+\left|z_{-}\right|^{2}\left|a_{2}\right|^{2}\right] \\
C_{k}(t) & =2 e^{-\pi \tau^{\prime} / 8}\left[\left|\operatorname{Re}\left(z_{+}^{*} z_{-}^{2} a_{6}\right)\right|+\left|\operatorname{Re}\left(z_{+}^{2} z_{-}^{*} a_{7}^{*}\right)\right|\right]+2 e^{-\pi \tau^{\prime} / 4}\left[\left|\operatorname{Re}\left(\left|z_{+}\right|^{2} z_{-} a_{1}^{*} a_{3}\right)\right|+\left|\operatorname{Re}\left(\left|z_{-}\right|^{2} z_{+} a_{2} a_{3}^{*}\right)\right|\right. \\
& \left.+\left|\operatorname{Re}\left(z_{+}^{*} z_{+}^{2} a_{1} a_{4} *\right)\right|+\left|\operatorname{Re}\left(z_{+}^{*}\left(z_{-}^{*}\right)^{2} a_{1} a_{5}^{*}\right)\right|+\left|\operatorname{Re}\left(z_{-} z_{+}^{2} a_{2} a_{4}^{*}\right)\right|+\left|\operatorname{Re}\left(z_{-}\left(z_{-} *\right)^{2} a_{2} a_{5}^{*}\right)\right|\right] \\
D_{k}(t) & =e^{-\pi \tau^{\prime} / 4}\left[\left|z_{+}\right|^{4}\left|a_{4}\right|^{2}+\left|z_{-}\right|^{4}\left|a_{5}\right|^{2}+\left|z_{+}\right|^{2}\left|z_{-}\right|^{2}\left|a_{3}\right|^{2}\right]+2 e^{-\pi \tau^{\prime} / 4}\left[\left|\operatorname{Re}\left(\left(z_{+}^{*}\right)^{2}\left(z_{-}^{*}\right)^{2} a_{4} a_{5}^{*}\right)\right|\right. \\
& +\left|\operatorname{Re}\left(\left|z_{+}\right|^{2}\left(z_{-}^{*}\right)^{2} a_{1} a_{6}^{*}\right)\right|+\left|\operatorname{Re}\left(z_{+} z_{-}^{*}\left|z_{+}\right|^{2} a_{1} a_{7}^{*}\right)\right|+\left|\operatorname{Re}\left(z_{+} z_{-}^{*}\left|z_{-}\right|^{2} a_{2} a_{6}^{*}\right)\right|+\left|\operatorname{Re}\left(z_{+}^{*} z_{-}^{*}\left|z_{-}\right|^{2} a_{3} a_{5}^{*}\right)\right| \\
& \left.+\left|\operatorname{Re}\left(z_{+}^{2}\left|z_{-}\right|^{2} a_{2} a_{7}^{*}\right)\right|+\left|\operatorname{Re}\left(z_{+} z_{-}\left|z_{+}\right|^{2} a_{3} a_{4}^{*}\right)\right|\right] .
\end{aligned}
$$

The necessary algebra leads us to the conclusion that $A_{k}(t)=0$, which establishes the fact that there is no linear $\delta$ term present in $F_{k}(t)$. One can get the follwing contribution from $B_{k}(t)$

$$
B_{k}(t) \simeq-\delta^{2}\left[2 \tau \tau^{\prime} e^{-\pi \tau^{\prime} / 2}+3 \tau \tau^{\prime} e^{-\pi \tau^{\prime} / 4}\right]-t^{2}\left[\frac{2 \tau^{\prime}}{\tau} e^{-\pi \tau^{\prime} / 2}+\frac{3 \tau^{\prime}}{\tau} e^{-\pi \tau^{\prime} / 4}\right]
$$

Similarly, $C_{k}(t)$ gives $O\left(z_{ \pm}^{3}\right)$ term,

$$
\begin{gathered}
C_{k}(t) \simeq-\delta^{2} t \tau^{1 / 2} e^{-\pi \tau^{\prime} / 8}\left[\frac{96 \pi \tau^{\prime 2}}{64\left|\Gamma\left(\frac{1}{2}+\frac{n^{*}}{2}\right) \Gamma\left(1+\frac{n}{2}\right)\right|}-\frac{96 \pi \tau^{\prime}}{8\left|\Gamma\left(\frac{1}{2}+\frac{n}{2}\right) \Gamma\left(1+\frac{n^{*}}{2}\right)\right|}\right]-\frac{40 \delta^{2} \pi t \tau^{1 / 2} \tau^{\prime} e^{-3 \pi \tau^{\prime} / 8}}{3\left|\Gamma\left(\frac{1}{2}+\frac{n^{*}}{2}\right) \Gamma\left(1+\frac{n}{2}\right)\right|} \\
+\quad t^{3} \tau^{-3 / 2} e^{-\pi \tau^{\prime} / 8}\left[\frac{96 \pi \tau^{\prime 2}}{64\left|\Gamma\left(\frac{1}{2}+\frac{n^{*}}{2}\right) \Gamma\left(1+\frac{n}{2}\right)\right|}-\frac{6 \pi \tau^{\prime}}{\left|\Gamma\left(\frac{1}{2}+\frac{n}{2}\right) \Gamma\left(1+\frac{n^{*}}{2}\right)\right|}\right]-\frac{5 t^{3} \tau^{-3 / 2} \pi \tau^{\prime} e^{-3 \pi \tau^{\prime} / 8}}{\left|\Gamma\left(\frac{1}{2}+\frac{n}{2}\right) \Gamma\left(1+\frac{n^{*}}{2}\right)\right|}
\end{gathered}
$$

The fourth order term in $z_{ \pm}$is given by $D_{k}(t)$,

$$
\begin{aligned}
& D_{k}(t) \simeq-\delta^{2} t^{2}\left[e^{-\pi \tau^{\prime} / 2}\left(\alpha_{1}+\beta_{1} \tau^{\prime 2}\right)+e^{-\pi \tau^{\prime} / 4} \times\left(\alpha_{2}+\beta_{2} \tau^{\prime 2}\right)\right]-t^{4}\left[\frac{e^{-\pi \tau^{\prime} / 2}}{\tau^{2}}\left(\alpha_{3}+\eta_{1} \tau^{\prime}+\beta_{3} \tau^{\prime 2}\right)\right. \\
& \left.+\quad \frac{e^{-\pi \tau^{\prime} / 4}}{\tau^{2}}\left(\alpha_{4}+\eta_{2} \tau^{\prime}+\beta_{4} \tau^{\prime 2}\right)\right]-\delta^{4}\left[e^{-\pi \tau^{\prime} / 2} \tau^{2}\left(\alpha_{5}+\eta_{3} \tau^{\prime}+\beta_{5} \tau^{\prime 2}\right)+e^{-\pi \tau^{\prime} / 4} \tau^{2}\left(\alpha_{6}+\eta_{4} \tau^{\prime}+\beta_{6} \tau^{\prime 2}\right) \mathrm{s} 21\right)
\end{aligned}
$$

where $\alpha$ 's, $\beta$ 's and $\eta$ 's are the numbers coming from the $\Gamma$-function. 
Using the approximation of $\ln (1+x) \simeq x$ for $x \ll 1$ and expanding near the critical point $h=1$ and the critical mode $k=0$, we can now compute the DF by evaluating the following integral over momentum space:

$$
\begin{aligned}
& \int_{0}^{\infty} d k \ln F_{k}(t) \simeq-\delta^{2}\left(\alpha \tau^{1 / 2}+\beta t+\eta t^{2} \tau^{-1 / 2}\right)-\phi t^{2} \tau^{-3 / 2}-\rho t^{3} \tau^{-2}-\gamma t^{4} \tau^{-5 / 2}-\theta \delta^{4} \tau^{3 / 2} \\
& \simeq \quad-\delta^{2}\left(\alpha \tau^{1 / 2}+\beta t+\eta t^{2} \tau^{-1 / 2}\right)+\cdots
\end{aligned}
$$

In the last line we have included only the leading order contributions to the integral. Here, $\alpha, \beta, \cdots$ are the numerical prefactors coming from the integartion of the dimensionless $\Gamma$-functions and the dimensionless exponentials.

The DF in the limit $t=\epsilon, \epsilon \rightarrow+0$, is therefore given by

$$
\begin{aligned}
D(t) & \approx \exp \left(\frac{L}{2 \pi} \int_{0}^{\pi} d k \ln F_{k}(t)\right) \\
& \simeq \exp \left(-\frac{\delta^{2} L}{2 \pi}\left[\alpha \tau^{1 / 2}+\beta t+\eta t^{2} \tau^{-1 / 2}\right]\right) \\
& \simeq 1-\frac{\delta^{2} L}{2 \pi}\left(\alpha \tau^{1 / 2}+\beta t+\eta t^{2} \tau^{-1 / 2}\right)
\end{aligned}
$$

Clearly, one finds that $\chi_{F}(\tau) \sim \tau^{1 / 2}, \alpha_{1}(\tau) \sim \tau^{0}$ and $\alpha_{2}(\tau) \sim \tau^{-1 / 2}$ as predicted by dimensional analysis argument given in Eq. (6).

Referring to the Fig. 1 of the main text, we consider now the case when $\gamma=J_{x}-J_{y}$ is ramped as $\gamma=-t / \tau$ with $J_{x}+J_{y}=h=1$ fixed in Eq. (S4). In this case, one has a ramp along the gapless Ising critical line across the MCP. Using the notion of the dominant critical point and exponents $d=\nu=1$ and $z=2[\mathrm{~S} 3$, 4], Eq. (4) yields

$$
\chi_{F}(\tau) \sim \tau^{1 / 3}, \quad \alpha_{1}(\tau) \sim \tau^{-1 / 3}, \quad \alpha_{2}(\tau) \sim \tau^{-1}
$$

for the linear ramp (see Fig. S1(a) ) while

$$
\chi_{F}(\tau) \sim \tau^{r /(2 r+1)}, \quad \alpha_{1}(\tau) \sim \tau^{-r /(2 r+1)}, \quad \alpha_{2}(\tau) \sim \tau^{-3 r /(2 r+1)}
$$

for the non-linear ramp (see Fig. S1 (b)) .

We shall verify the above scaling form of $\chi_{F}(\tau), \alpha_{1}(\tau)$ and $\alpha_{2}(\tau)$ by explicitly calculating the DF. The interaction Hamiltonian between the environment and the qubit is accordingly modified as $H_{S E}=-(\delta / 2) \sum_{i}\left(\sigma_{i}^{x} \sigma_{i+1}^{x}-\sigma_{i}^{y} \sigma_{i+1}^{y}\right) \sigma_{S}^{z}$. The coupling $\delta$ therefore provides two channels of the temporal evolution of the environmental ground state with anisotropy $\gamma+\delta$ and $\gamma-\delta$. The time-dependent Bogoliubov-de Gennes equation governing the time evolution of environmental state through the gapless line are given by

$$
\begin{aligned}
i \frac{d}{d t} u_{k}^{ \pm} & =2(\gamma(t) \mp \delta) \sin k u_{k}^{ \pm}+2(h-\cos k) v_{k}^{ \pm}, \\
i \frac{d}{d t} v_{k}^{ \pm} & =2(h-\cos k) u_{k}^{ \pm}-2(\gamma(t) \mp \delta) \sin k v_{k}^{ \pm} .
\end{aligned}
$$

Accordingly, we use the notation, $t_{ \pm}^{\prime}=4 \tau(h-\cos k)(\gamma(t) \mp \delta), \tau^{\prime}=4 \tau(h-\cos k)^{2} / \sin k, z_{ \pm}=t_{ \pm}^{\prime} e^{i \pi / 4} / \sqrt{\tau^{\prime}}$ and $n=-i \tau^{\prime} / 4$.

The solutions are the same as obtained for the case of transverse field quenching given in Eq. (S11) and Eq. (S12). In order to estimate $F_{k}(t)$ with $t=\epsilon, \epsilon \rightarrow+0$, one has to use the following approximation: $t_{+}^{\prime} \ll \sqrt{ } \tau^{\prime}$ and $\delta^{2} \tau k \ll 1$. Following the same line of algebra one can find out a similar expression of $u_{k}^{+*}(t) u_{k}^{-}(t)+v_{k}^{+*}(t) v_{k}^{-}(t)$ with a modified $z_{ \pm}^{\text {gapless }}=2 \sqrt{\tau \sin k}(t / \tau \mp \delta) \exp (i \pi / 4)$. One can note that for the Ising model $z_{ \pm}^{\text {Ising }}=2 \sqrt{\tau}(t / \tau \mp \delta) \exp (i \pi / 4)$. As a result, $z_{ \pm}^{\text {gapless }}=\sqrt{\sin k} z_{ \pm}^{\text {Ising }}$. In this case, we find $A_{k}(t)=0$. The $O\left(z_{ \pm}^{2}\right)$ contribution is given by $B_{k}^{\text {gapless }}=$ $\sin k B_{k}^{\text {Ising }}$. Similarly, $C_{k}^{\text {gapless }}=\sin ^{3 / 2} k C_{k}^{\text {Ising }}$ and $D_{k}^{\text {gapless }}=\sin ^{2} k D_{k}^{\text {Ising }}$.

One can therefore calculate the integral of $F_{k}(t)$ by using the logarithmic approximation and expanding momentum near the critical mode, $\sin k \simeq k$ and $\tau^{\prime} \simeq k^{3} \tau$

$$
\begin{aligned}
& \int_{0}^{\infty} d k \ln F_{k}(t) \simeq-\delta^{2}\left(\alpha \tau^{1 / 3}+\beta t \tau^{-1 / 3}+\eta t^{2} \tau^{-1}\right)-\phi t^{2} \tau^{-5 / 3}-\rho t^{3} \tau^{-7 / 3}-\gamma t^{4} \tau^{-3}-\theta \delta^{4} \tau \\
& \simeq \quad-\delta^{2}\left(\alpha \tau^{1 / 3}+\beta t \tau^{-1 / 3}+\eta t^{2} \tau^{-1}\right)+\cdots
\end{aligned}
$$

The DF $D(t)$ in the vicinity of MCP at $t=\epsilon, \epsilon \rightarrow+0$ is given by

$$
D(t) \simeq 1-\frac{\delta^{2} L}{2 \pi}\left(\alpha \tau^{1 / 3}+\beta t \tau^{-1 / 3}+\eta t^{2} \tau^{-1}\right)
$$




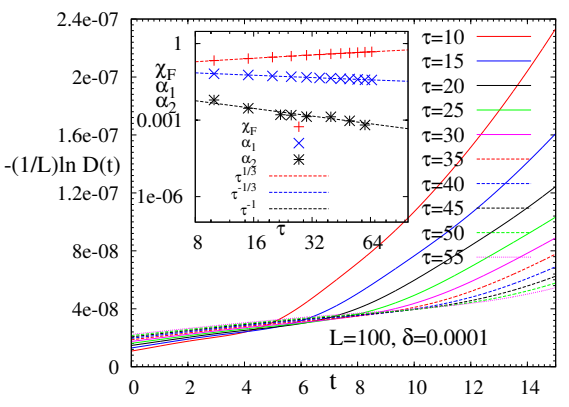

(a)

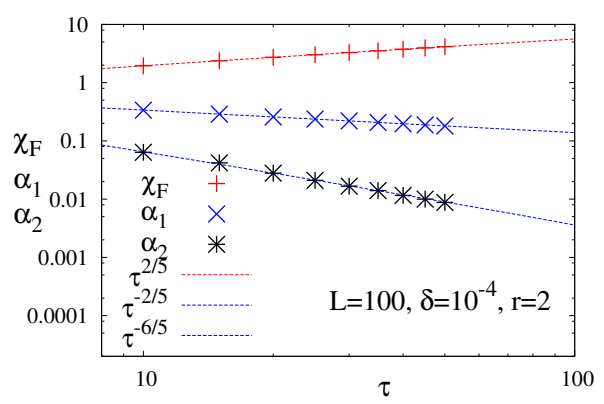

(b)

FIG. S1: (a) Early-time evolution of the decoherence factor for quenching the parameter $\gamma$ as $-t / \tau$ of the Hamiltonian 1 . with the transverse field is fixed at $h=1$; the left panel shows scaling of $\chi_{L}, \alpha_{1}$ and $\alpha_{2}$. Results are in good agreement with the prediction in Eq. (S24). (b) Numerical results confirm the scaling for the non-linear case given in Eq. (S25).

This expression of $D(t)$ clearly verifies the scaling of $\chi_{F}(\tau), \alpha_{1}(\tau)$ and $\alpha_{2}(\tau)$ predicted above in Eq. (S24).

On the other hand, for an anisotropic quenching when $\gamma$ is quenched with $|h|<1$ and the gap vanishes for the critical mode $k_{c}=\cos ^{-1} h$, we have $\tau^{\prime} \simeq 4 \tau \sqrt{1-h^{2}} k^{2}$ and $\sin k \simeq \sqrt{1-h^{2}}$; using these parametrizations, one can retrieve scaling $\chi_{F}(\tau), \alpha_{1}(\tau)$ and $\alpha_{2}(\tau)$ similar to those obtained in the $h$-quenching case.

\section{INTEGRABLE MODELS: SCALING OF $\ln D(t)$ FAR-AWAY FROM THE CRITICAL POINT $(t \gg 0)$}

Let us now address the issue that what happens to the DF when the system is quenched far away from the QCP, i.e., $h$ in model (1) is changed from a large negative to a large positive value. For an integrable model reducible to decoupled two-level problems (like the model (1) or the Kitaev honeycomb model [S5]), one can analytically establish that in the limit $t \gg 0, \ln D \sim\left(-t^{2} \delta^{2} L^{d} \tilde{\alpha}_{2}(\tau)\right)$, i.e., there is a prominent Gaussian decay with time [S2, 6]. Damski et al. [S2], proposed that for an integrable ESS, $\tilde{\alpha}_{2}(\tau)$ is likely to scale as $n$ as determined by the KZS relation. In a subsequent work, Nag et al. [ $[\underline{6}$ ] showed that this conjecture is not necessarily true even for the above integrable model when the system is quenched along a gapless critical line of the model (1); one finds $n \sim \tau^{-1 / 3}$ while on the contrary $\tilde{\alpha}_{2}(\tau) \sim \tau^{-1}$. Using the dimensional analysis argument presented above, we immediately conclude that $\tilde{\alpha}_{2}(\tau) \sim \tau^{(2-d \nu-2 z \nu) /(z \nu+1)}$ for a linear quenching of $\lambda$ while for a non-linear ramp $\tilde{\alpha}_{2}(\tau) \sim \tau^{r(2-d \nu-2 z \nu) /(r z \nu+1)}$. It is noteworthy that whenever $\nu z=1$, the scaling of $\tilde{\alpha}_{2}$ is the same as that of $n$. This holds true even for the non-linear ramp where both $\tilde{\alpha}_{2}$ and $n$ scale as $\tau^{-\frac{r \nu d}{(r \nu z+1)}}$. On the other hand, when the environmental XY chain is quenched along the gapless Ising critical line $h=1$ (varying $\gamma=t / \tau$ across the multi-critical point at $h=1, \gamma=0$ ), one can argue that the multi-critical point plays the role of a dominant critical point with critical exponents that $\nu=1$ and $z=2\left[\mathrm{~S} 3\right.$ ] and hence $\nu z \neq 1$; substituting these exponents in the KZS relation and the predicted scaling $\tilde{\alpha}_{2}$, one immediately finds $n \sim \tau^{-1 / 3}$ and $\tilde{\alpha} \sim \tau^{-1}$. Therefore, the universal scaling formula of $\tilde{\alpha}_{2}(\tau)$ explains all the integrable situations discussed in earlier studies.

[S1] L. C. Venuti and P. Zanardi, Phys. Rev. Lett. 99, 095701 (2007). P. Zanardi, P. Giorda, and M. Cozzini, Phys. Rev. Lett. 99, 100603 (2007); D. Schwandt, F. Alet, and S. Capponi, Phys. Rev. Lett. 103, 170501 (2009). C. De Grandi, V. Gritsev, and A. Polkovnikov, Phys. Rev. B 81, 012303 (2010); S.-J. Gu, Int. J. Mod. Phys B 24, 4371 (2010); V. Mukherjee, A. Polkovnikov, and A. Dutta, Phys. Rev. B 83, 075118 (2011); M. M. Rams and B. Damski, Phys. Rev. Lett. 106, 055701 (2011); V. Mukherjee and A. Dutta, Phys. Rev. B 83, 214302 (2011); V. Mukherjee, A. Dutta, D. Sen, Phys. Rev. B 85, 024301 (2012).

[S2] B. Damski, H. T. Quan, and W. H. Zurek, Phys. Rev. B 83, 062104 (2011).

[S3] S.Deng, G. Ortiz, L. Viola EPL, 84, 67008 (2008).

[S4] U. Divakaran, A. Dutta, and D. Sen, Phys. Rev. B 78, 144301 (2008).

[S5] A. Kitaev, Ann. Phys. 321, 2 (2006).

[S6] T. Nag, U. Divakaran and A. Dutta, Phys. Rev. B 86, 020401 (R) (2012). 